\title{
Dehydration in harsh winter from dry heat: Case series
}

\author{
Samiksha Gupta, Moises Auron and Mohit Gupta* \\ Department of Internal Medicine, Cleveland clinic, Ohio, USA
}

\begin{abstract}
Dehydration occurs when the loss of body fluids exceeds the intake of water. Although dehydration occurs most often during the summer months, clinicians require to have increased suspicion of its potential to occur even during the cold winter months as patients are exposed to increased indoor dry heat. A peculiar phenomenon in dry environments is the development of increased static electricity, which can be a warning sign of the degree of dryness in a household and prompt the individual to enhance their fluid intake. We aim to create awareness of this phenomenon and also to increase sensibility around the risk for dehydration in the winter.
\end{abstract}

Herein, we report two cases of inpatient admissions with dehydration and acute renal injury during the winter months in Northeast Ohio. A unique feature common to both patients was the presence of increased static electricity in both patients' households in association with decreased relative humidity.

\section{Cases}

\section{Case 1}

A 50 year-old woman, teacher by profession, healthy, presented with 2 days of progressively worsening malaise, nausea, postural dizziness and dark urine. Her exam showed BP 80/51 mmHg, heart rate of $130 \mathrm{bpm}$; her mentation was normal; mucous membranes were dry, jugular vein was flat; cardiac exam revealed tachycardia, but no gallop. Pulses were very faint and capillary refill was prolonged (3 seconds). Diagnostic testing showed creatinine $1.4 \mathrm{mg} / \mathrm{dL}$ (baseline $0.8 \mathrm{mg} / \mathrm{dL}$ ). She denied any volume losses or change in water intake. Further history revealed that she has been staying indoor all the time, and that her home furnace provides dry heat as the humidifier is not functional. She stated that a common phenomenon was the presence of substantial static electricity over all her household since the beginning of the winter season (and hence, using the home furnace).

\section{Case 2}

A 82 year-old woman with longstanding history of hypertension (on Lisinopril) and hyperlipidemia (on statin) who lives alone and is baseline well-functional, presented to routine preventive health appointment during the winter months, where routine diagnostic tests revealed creatinine of $2.1 \mathrm{mg} / \mathrm{dL}$ (baseline $1.2 \mathrm{mg} / \mathrm{dL}$ ). Her exam showed, normal mentation, dry mucous membranes and prolonged capillary refill $(3 \mathrm{sec})$. She denied any new symptoms, as well as any changes in medications, fluid intake, or volume losses. She stated that she spends most of the time indoors, and her house is heated by a furnace, with no humidifier. When asked, she endorsed as well the presence of significant static electricity phenomenon over all her household too, which has also been occurring with temporal correlation with the beginning of the winter season.

\section{Discussion}

Dehydration occurs when the fluid intake is unable to match the body fluid losses in form of sensible and insensible perspiration. Dehydration can manifests as non-specific symptoms depending on its severity. Mild or moderate dehydration presents as increased thirst, dry mouth, dry skin, headache, muscle cramps, and dizziness. If unattended, patients can manifest with features of severe dehydration that include decreased organ perfusion leading to oliguria/anuria, hypotension, tachycardia, confusion, or coma [1].

There are a multitude of factors responsible for dehydration in winters including cold induced diuresis, decreased intake of water, winter clothing, increased respiratory losses and skin losses [2]. Evaporation of fluid from the skin surface in the case of sweating, or from moist membranes of the respiratory tract is dependent on the heat index of environment which is governed by two factors, air temperature and relative humidity [3]. The loss of body fluid is more in dry warm air when the temperature is high and the relative humidity is less. During the winter months, there is a reduction in relative humidity due to heating the air to a comfortable working temperature, which predisposes an individual to dehydration. In addition, decrease in relative humidity during winters, also increases the chance of production of static electricity.

Herein, we report two patients who presented in the winter months with dehydration and hypovolemia. Both reported using central heating but without any humidification. The unique aspect in cases was that both of them also reported of prominent static electricity phenomenon at their households.

\section{Authorship and Contributor ship}

Authors: Samiksha Gupta, Moises Auron and Mohit Gupta

Contributors: None

\section{Acknowledgments}

None.

${ }^{*}$ Correspondence to: Mohit Gupta, Department of Internal Medicine, Cleveland clinic, Ohio-44195, USA, Tel: +1(216)401-6104; E-mail: GUPTAM32@ccf.org

Key words: static electricity, Dehydration, Winter, decreased relative humidity

Received: July 18, 2018; Accepted: July 26, 2018; Published: July 28, 2018 


\section{Funding information}

NA.

\section{Competing interest}

None.

\section{References}

1. Marriott BM, Carlson SJ (1996) Nutritional Needs In Cold And In High-Altitude Environments: Applications for Military Personnel in Field Operations. [e-book] Washington (DC): National Academies Press (US).

2. Marriott BM, Carlson SJ (1996) Influence of Cold Stress on Human Fluid Balance. National Academies Press (US) Washington (DC).

3. Occupational Safety and Health Administration (2018) Using the Heat Index: A Guide for Employers, Washington.

Copyright: $(\mathbb{O} 2018$ Gupta S. This is an open-access article distributed under the terms of the Creative Commons Attribution License, which permits unrestricted use, distribution, and reproduction in any medium, provided the original author and source are credited. 